\title{
International bureaucracies and their influence on policy-making: a review of empirical evidence (Steffen Eckhard and Jörn Ege)
}

Online Appendix A: Specific search terms used to identify the core sample

To identify bureaucracy-centered IO research, the common occurrence of the keywords 'international (governmental) organization' and 'bureaucracy' (using different truncations and wildcards) was used to search title, abstract/ summary and the subject keyword section of the two databases. To extract publications with a distinct focus on international public administration, various forms of the terms 'international bureaucracy', 'international (public) administration', 'international civil service' and 'international secretariat' were used. Because the WorldCat database reported too many irrelevant hits, we had to combine these keywords with the term 'international (governmental) organization' to further specify the search parameter there.

\begin{tabular}{|c|c|c|c|}
\hline Database & focus on & Keywords & further restrictions \\
\hline \multirow[t]{2}{*}{$\begin{array}{l}\text { Web of } \\
\text { Science } \\
\text { (article } \\
\text { search) }\end{array}$} & $\begin{array}{l}\text { International } \\
\text { Public } \\
\text { Administration }\end{array}$ & $\begin{array}{l}\text { TOPIC: ("international bureaucra*") } O R \text { ("international } \\
\text { administrat*") } O R \text { ("international public administrat*”) } O R \\
\text { ("transnational administrat*") OR ("transnational public } \\
\text { administrat*") } O R \text { ("supranational administrat*”) } O R \\
\text { ("international civil serv*") } O R \text { ("international secretar*") }\end{array}$ & $\begin{array}{l}\text { Research domains: Social } \\
\text { Science } \\
\text { Languages: English } \\
\text { Document types: articles }\end{array}$ \\
\hline & $\begin{array}{l}\text { International } \\
\text { Organization }\end{array}$ & $\begin{array}{l}\text { TOPIC: ((“international organi?ation*”) AND (“bureaucra*”)) } \\
\text { OR ((“intergovernmental organi?ation*”) AND (“bureaucra*”)) } \\
\text { OR ((“international governmental organi?ation*”) AND } \\
\text { ("bureaucra*”)) }\end{array}$ & $\begin{array}{l}\text { Research domains: Social } \\
\text { Science } \\
\text { Languages: English } \\
\text { Document types: articles }\end{array}$ \\
\hline \multirow[t]{2}{*}{$\begin{array}{l}\text { WorldCat } \\
\text { (book } \\
\text { search) }\end{array}$} & $\begin{array}{l}\text { International } \\
\text { Public } \\
\text { Administration }\end{array}$ & 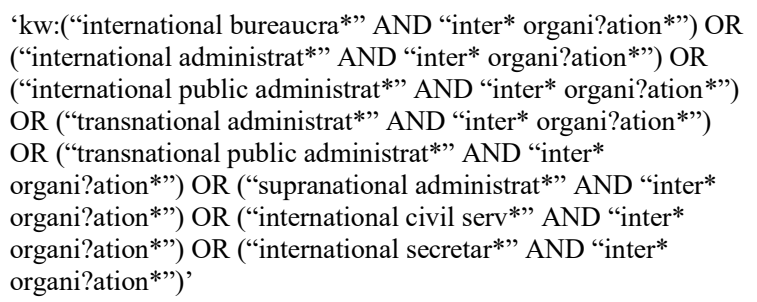 & $\begin{array}{l}\text { 'Book'> 'Non-Fiction'> } \\
\text { 'English' > 'Non-Juvenile' }\end{array}$ \\
\hline & $\begin{array}{l}\text { International } \\
\text { Organization }\end{array}$ & $\begin{array}{l}\text { 'kw:(“international organi?ation*” AND “bureaucra*”) OR } \\
\text { ("intergovernmental organi?ation*” AND "bureaucra*”) OR } \\
\text { ("international governmental organi?ation"” AND "bureaucra*”), }\end{array}$ & $\begin{array}{l}>\text { 'Book' > 'Non-Fiction' > } \\
\text { 'English'> 'Non-Juvenile' }\end{array}$ \\
\hline
\end{tabular}


Online Appendix B: List of publications in the sample along the four levels of research (in alphabetical order)

\begin{tabular}{|c|c|c|c|c|c|c|}
\hline author & year & title & $\begin{array}{l}\text { journal/ } \\
\text { publisher }\end{array}$ & primary focus & IOs studied & $\begin{array}{l}\text { disciplinary } \\
\text { background }\end{array}$ \\
\hline $\begin{array}{l}\text { Barnett, M.N. and } \\
\text { Finnemore, M. }\end{array}$ & 1999 & $\begin{array}{l}\text { 'The politics, power, and } \\
\text { pathologies of international } \\
\text { organizations' }\end{array}$ & $\begin{array}{l}\text { International } \\
\text { Organization } \\
\text { 53(4): 699-732. }\end{array}$ & $\begin{array}{l}\text { policy } \\
\text { formulation }\end{array}$ & $\begin{array}{l}\text { examples } \\
\text { from UN, } \\
\text { UNHCR, } \\
\text { World Bank, } \\
\text { IMF, OSCE, } \\
\text { EU }\end{array}$ & $\begin{array}{l}\text { international } \\
\text { relations }\end{array}$ \\
\hline $\begin{array}{l}\text { Barnett, M.N. and } \\
\text { Finnemore, M. }\end{array}$ & 2004 & $\begin{array}{l}\text { Rules for the World: International } \\
\text { Organizations in Global Politics }\end{array}$ & $\begin{array}{l}\text { Ithaca (NY): } \\
\text { Cornell University } \\
\text { Press. }\end{array}$ & $\begin{array}{l}\text { policy } \\
\text { formulation }\end{array}$ & $\begin{array}{l}\text { UN, UNHCR, } \\
\text { IMF }\end{array}$ & $\begin{array}{l}\text { international } \\
\text { relations }\end{array}$ \\
\hline Bauer, M.W. & 2012 & $\begin{array}{l}\text { 'Tolerant if personal goals remain } \\
\text { unharmed: explaining } \\
\text { supranational bureaucrats' } \\
\text { attitudes to organizational change' }\end{array}$ & $\begin{array}{l}\text { Governance 25(3): } \\
485-510 .\end{array}$ & individual level & $\mathrm{EU}$ & $\begin{array}{l}\text { public } \\
\text { administration }\end{array}$ \\
\hline Benedek, W. & 2010 & $\begin{array}{l}\text { "Lessons (not) Learned" with } \\
\text { Regard to Human Rights and } \\
\text { Democracy: A Comparison of } \\
\text { Bosnia and Herzegovina, Kosovo } \\
\text { and Macedonia }\end{array}$ & $\begin{array}{l}\text { Wien: Neuer } \\
\text { Wissenschaftlicher } \\
\text { Verlag. }\end{array}$ & $\begin{array}{l}\text { policy } \\
\text { implementation }\end{array}$ & $\mathrm{n}>4$ & $\begin{array}{l}\text { international } \\
\text { relations }\end{array}$ \\
\hline $\begin{array}{l}\text { Benner, T., } \\
\text { Mergenthaler, S. } \\
\text { and Rotmann, P. }\end{array}$ & 2011 & $\begin{array}{l}\text { The New World of UN Peace } \\
\text { Operations: Learning to Build } \\
\text { Peace }\end{array}$ & $\begin{array}{l}\text { Oxford: Oxford } \\
\text { University Press. }\end{array}$ & $\begin{array}{l}\text { policy } \\
\text { formulation }\end{array}$ & UN & $\begin{array}{l}\text { international } \\
\text { relations }\end{array}$ \\
\hline Best, J. & 2012 & 'Bureaucratic ambiguity' & $\begin{array}{l}\text { Economy and } \\
\text { Society 41(1): } 84- \\
106 .\end{array}$ & $\begin{array}{l}\text { policy } \\
\text { formulation }\end{array}$ & $\begin{array}{l}\text { IMF, World } \\
\text { Bank }\end{array}$ & other discipline \\
\hline $\begin{array}{l}\text { Bhattacharya, } \\
\text { A.K. }\end{array}$ & 1976 & $\begin{array}{l}\text { 'The influence of the international } \\
\text { secretariat: UNCTAD and } \\
\text { generalized tariff preferences' }\end{array}$ & $\begin{array}{l}\text { International } \\
\text { Organization } \\
\text { 30(1): } 75-90 .\end{array}$ & $\begin{array}{l}\text { policy } \\
\text { implementation }\end{array}$ & UNCTAD & $\begin{array}{l}\text { international } \\
\text { relations }\end{array}$ \\
\hline $\begin{array}{l}\text { Biermann, F. and } \\
\text { Siebenhüner, B. } \\
\text { (eds) }\end{array}$ & 2009 & $\begin{array}{l}\text { Managers of Global Change: The } \\
\text { Influence of International } \\
\text { Environmental Bureaucracies }\end{array}$ & $\begin{array}{l}\text { Cambridge (MA): } \\
\text { The MIT Press. }\end{array}$ & $\begin{array}{l}\text { policy } \\
\text { implementation }\end{array}$ & $\mathrm{n}>4$ & $\begin{array}{l}\text { public } \\
\text { administration }\end{array}$ \\
\hline Blom-Hansen, J. & 2008 & $\begin{array}{l}\text { 'The origins of the EU comitology } \\
\text { system: a case of informal agenda } \\
\text { setting by the commission' }\end{array}$ & $\begin{array}{l}\text { Journal of } \\
\text { European Public } \\
\text { Policy 15(2): 208- } \\
26 .\end{array}$ & $\begin{array}{l}\text { institutional } \\
\text { design }\end{array}$ & EU & $\begin{array}{l}\text { public } \\
\text { administration }\end{array}$ \\
\hline Bohne, E. & 2010 & $\begin{array}{l}\text { The World Trade Organization: } \\
\text { Institutional Development and } \\
\text { Reform }\end{array}$ & $\begin{array}{l}\text { Basingstoke: } \\
\text { Palgrave } \\
\text { Macmillan. }\end{array}$ & $\begin{array}{l}\text { policy } \\
\text { implementation }\end{array}$ & WTO & $\begin{array}{l}\text { public } \\
\text { administration }\end{array}$ \\
\hline Brandsma, G.J. & 2012 & $\begin{array}{l}\text { 'The effect of information on } \\
\text { oversight: the European } \\
\text { Parliament's response to } \\
\text { increasing information on } \\
\text { comitology decision-making' }\end{array}$ & $\begin{array}{l}\text { International } \\
\text { Review of } \\
\text { Administrative } \\
\text { Sciences } 78(1) \text { : } \\
74-92 .\end{array}$ & $\begin{array}{l}\text { institutional } \\
\text { design }\end{array}$ & $\mathrm{EU}$ & $\begin{array}{l}\text { public } \\
\text { administration }\end{array}$ \\
\hline Broome, A. & 2012 & $\begin{array}{l}\text { 'The politics of IMF-EU } \\
\text { cooperation: institutional change } \\
\text { from the Maastricht treaty to the } \\
\text { launch of the Euro' }\end{array}$ & $\begin{array}{l}\text { Journal of } \\
\text { European Public } \\
\text { Policy 20(4): 589- } \\
604 .\end{array}$ & $\begin{array}{l}\text { institutional } \\
\text { design }\end{array}$ & IMF & $\begin{array}{l}\text { public } \\
\text { administration }\end{array}$ \\
\hline Caplan, R. & 2002 & $\begin{array}{l}\text { A New Trusteeship? The } \\
\text { International Administration of } \\
\text { War-Torn Territories }\end{array}$ & $\begin{array}{l}\text { London: } \\
\text { Routledge. }\end{array}$ & $\begin{array}{l}\text { policy } \\
\text { implementation }\end{array}$ & EU, UN & $\begin{array}{l}\text { international } \\
\text { relations }\end{array}$ \\
\hline
\end{tabular}




\begin{tabular}{|c|c|c|c|c|c|c|}
\hline Chorev, N. & 2013 & $\begin{array}{l}\text { 'Restructuring Neoliberalism at } \\
\text { the World Health Organization' }\end{array}$ & $\begin{array}{l}\text { Review of } \\
\text { International } \\
\text { Political Economy } \\
\text { 20(4): 627-66. }\end{array}$ & $\begin{array}{l}\text { policy } \\
\text { implementation }\end{array}$ & WHO & $\begin{array}{l}\text { international } \\
\text { relations }\end{array}$ \\
\hline Chorev, N. & 2012 & $\begin{array}{l}\text { The World Health Organization } \\
\text { between North and South }\end{array}$ & $\begin{array}{l}\text { Ithaca (NY): } \\
\text { Cornell University } \\
\text { Press. }\end{array}$ & $\begin{array}{l}\text { policy } \\
\text { formulation }\end{array}$ & WHO & $\begin{array}{l}\text { international } \\
\text { relations }\end{array}$ \\
\hline $\begin{array}{l}\text { Conceicão-Heldt, } \\
\text { E. d. }\end{array}$ & 2013 & $\begin{array}{l}\text { 'Do agents "run amok"? A } \\
\text { comparison of agency slack in the } \\
\text { EU and US trade policy in the } \\
\text { Doha Round' }\end{array}$ & $\begin{array}{l}\text { Journal of } \\
\text { Comparative } \\
\text { Policy Analysis } \\
\text { 15(1): 21-36. }\end{array}$ & $\begin{array}{l}\text { institutional } \\
\text { design }\end{array}$ & $\mathrm{EU}$ & $\begin{array}{l}\text { public } \\
\text { administration }\end{array}$ \\
\hline $\begin{array}{l}\text { Copelovitch, } \\
\text { M.S. }\end{array}$ & 2010 & $\begin{array}{l}\text { 'Master or servant? common } \\
\text { agency and the political economy } \\
\text { of IMF lending' }\end{array}$ & $\begin{array}{l}\text { International } \\
\text { Studies Quarterly } \\
\text { 54(1): 49-77. }\end{array}$ & $\begin{array}{l}\text { policy } \\
\text { formulation }\end{array}$ & IMF & $\begin{array}{l}\text { international } \\
\text { relations }\end{array}$ \\
\hline $\begin{array}{l}\text { Cox, R.W. and } \\
\text { Jacobson, H.K. } \\
\text { (eds) }\end{array}$ & 1973 & $\begin{array}{l}\text { The Anatomy of Influence: } \\
\text { Decision-Making in International } \\
\text { Organization }\end{array}$ & $\begin{array}{l}\text { New Haven }(\mathrm{CT}) \text { : } \\
\text { Yale University } \\
\text { Press. }\end{array}$ & $\begin{array}{l}\text { policy } \\
\text { implementation }\end{array}$ & $\mathrm{n}>4$ & $\begin{array}{l}\text { international } \\
\text { relations }\end{array}$ \\
\hline $\begin{array}{l}\text { Ellinas, A.A. and } \\
\text { Suleiman, E.N. }\end{array}$ & 2011 & $\begin{array}{l}\text { 'Supranationalism in a } \\
\text { transnational bureaucracy: the case } \\
\text { of the European Commission' }\end{array}$ & $\begin{array}{l}\text { Journal of } \\
\text { Common Market } \\
\text { Studies 49(5): } \\
923-47 .\end{array}$ & individual level & $\mathrm{EU}$ & $\begin{array}{l}\text { international } \\
\text { relations }\end{array}$ \\
\hline Elsig, M. & 2010 & $\begin{array}{l}\text { 'The World Trade Organization at } \\
\text { work: performance in a member- } \\
\text { driven milieu' }\end{array}$ & $\begin{array}{l}\text { The Review of } \\
\text { International } \\
\text { Organizations } \\
5(3): 345-63 \text {. }\end{array}$ & $\begin{array}{l}\text { policy } \\
\text { formulation }\end{array}$ & WTO & $\begin{array}{l}\text { international } \\
\text { relations }\end{array}$ \\
\hline Finnemore, M. & 1993 & $\begin{array}{l}\text { 'International organizations as } \\
\text { teachers of norms: the United } \\
\text { Nations educational, scientific, and } \\
\text { cultural organization and science } \\
\text { policy' }\end{array}$ & $\begin{array}{l}\text { International } \\
\text { Organization } \\
47(4): 565-97 .\end{array}$ & $\begin{array}{l}\text { policy } \\
\text { implementation }\end{array}$ & UNESCO & $\begin{array}{l}\text { international } \\
\text { relations }\end{array}$ \\
\hline Finnemore, M. & 1996 & $\begin{array}{l}\text { National Interests in International } \\
\text { Society }\end{array}$ & $\begin{array}{l}\text { Ithaca (NY): } \\
\text { Cornell University } \\
\text { Press. }\end{array}$ & $\begin{array}{l}\text { policy } \\
\text { implementation }\end{array}$ & $\begin{array}{l}\text { IRC, } \\
\text { UNESCO, } \\
\text { World Bank }\end{array}$ & $\begin{array}{l}\text { international } \\
\text { relations }\end{array}$ \\
\hline $\begin{array}{l}\text { Gest, N. and } \\
\text { Grigorescu, A. }\end{array}$ & 2010 & $\begin{array}{l}\text { 'Interactions among } \\
\text { intergovernmental organizations in } \\
\text { the anti-corruption realm' }\end{array}$ & $\begin{array}{l}\text { The Review of } \\
\text { International } \\
\text { Organizations } \\
\text { 5(1): 53-72. }\end{array}$ & $\begin{array}{l}\text { policy } \\
\text { implementation }\end{array}$ & $\mathrm{n}>4$ & $\begin{array}{l}\text { international } \\
\text { relations }\end{array}$ \\
\hline Goetz, K.H. & 2014 & $\begin{array}{l}\text { 'Time and power in the European } \\
\text { Commission' }\end{array}$ & $\begin{array}{l}\text { International } \\
\text { Review of } \\
\text { Administrative } \\
\text { Sciences } 80(3) \text { : } \\
577-96 .\end{array}$ & $\begin{array}{l}\text { institutional } \\
\text { design }\end{array}$ & EU & $\begin{array}{l}\text { public } \\
\text { administration }\end{array}$ \\
\hline Graham, E.R. & 2014 & $\begin{array}{l}\text { 'International organizations as } \\
\text { collective agents: fragmentation } \\
\text { and the limits of principal control } \\
\text { at the World Health Organization' }\end{array}$ & $\begin{array}{l}\text { European Journal } \\
\text { of International } \\
\text { Relations 20(2): } \\
366-90 .\end{array}$ & $\begin{array}{l}\text { policy } \\
\text { implementation }\end{array}$ & WHO & $\begin{array}{l}\text { international } \\
\text { relations }\end{array}$ \\
\hline Gravier, M. & 2013 & $\begin{array}{l}\text { 'Challenging or enhancing the } \\
\text { EU's legitimacy? the evolution of } \\
\text { representative bureaucracy in the } \\
\text { Commission's staff policy' }\end{array}$ & $\begin{array}{l}\text { Journal of Public } \\
\text { Administration } \\
\text { Research and } \\
\text { Theory 23(4): } 817- \\
\text { 38. }\end{array}$ & $\begin{array}{l}\text { institutional } \\
\text { design }\end{array}$ & EU & $\begin{array}{l}\text { public } \\
\text { administration }\end{array}$ \\
\hline Grigorescu, A. & 2010 & $\begin{array}{l}\text { 'The spread of bureaucratic } \\
\text { oversight mechanisms across } \\
\text { intergovernmental organizations' }\end{array}$ & $\begin{array}{l}\text { International } \\
\text { Studies Quarterly } \\
\text { 54(3): 871-86. }\end{array}$ & $\begin{array}{l}\text { institutional } \\
\text { design }\end{array}$ & $\mathrm{n}>4$ & $\begin{array}{l}\text { international } \\
\text { relations }\end{array}$ \\
\hline Hanrieder, $\mathrm{T}$. & 2014 & $\begin{array}{l}\text { 'Gradual change in international } \\
\text { organisations: agency theory and } \\
\text { historical institutionalism' }\end{array}$ & $\begin{array}{l}\text { Politics 34(4): } \\
\text { 324-33. }\end{array}$ & $\begin{array}{l}\text { institutional } \\
\text { design }\end{array}$ & $\begin{array}{l}\text { several } \\
\text { examples }\end{array}$ & $\begin{array}{l}\text { public } \\
\text { administration }\end{array}$ \\
\hline
\end{tabular}




\begin{tabular}{|c|c|c|c|c|c|c|}
\hline Harman, S. & 2011 & $\begin{array}{l}\text { 'Searching for an executive head? } \\
\text { leadership and UNAIDS' }\end{array}$ & $\begin{array}{l}\text { Global } \\
\text { Governance 17(4): } \\
\text { 429-46. }\end{array}$ & $\begin{array}{l}\text { institutional } \\
\text { design }\end{array}$ & UNAIDS & $\begin{array}{l}\text { international } \\
\text { relations }\end{array}$ \\
\hline Hirschmann, G. & 2012 & $\begin{array}{l}\text { 'Organizational learning in United } \\
\text { Nations' peacekeeping exit } \\
\text { strategies' }\end{array}$ & $\begin{array}{l}\text { Cooperation and } \\
\text { Conflict } 47(3): \\
368-85 .\end{array}$ & $\begin{array}{l}\text { policy } \\
\text { formulation }\end{array}$ & UN & $\begin{array}{l}\text { international } \\
\text { relations }\end{array}$ \\
\hline Jinnah, S. & 2011 & $\begin{array}{l}\text { 'Marketing linkages: secretariat } \\
\text { governance of the climate- } \\
\text { biodiversity interface' }\end{array}$ & $\begin{array}{l}\text { Global } \\
\text { Environmental } \\
\text { Politics 11(3): 23- } \\
43 .\end{array}$ & $\begin{array}{l}\text { policy } \\
\text { implementation }\end{array}$ & $\mathrm{CBD}$ & $\begin{array}{l}\text { public } \\
\text { administration }\end{array}$ \\
\hline Johns, L. & 2007 & $\begin{array}{l}\text { 'A servant of two masters: } \\
\text { communication and the selection } \\
\text { of international bureaucrats' }\end{array}$ & $\begin{array}{l}\text { International } \\
\text { Organization } \\
\text { 61(2): } 245-75 .\end{array}$ & individual level & UN & $\begin{array}{l}\text { international } \\
\text { relations }\end{array}$ \\
\hline Johnson, $\mathrm{T}$. & $2013 a$ & $\begin{array}{l}\text { 'Institutional design and } \\
\text { bureaucrats' impact on political } \\
\text { control' }\end{array}$ & $\begin{array}{l}\text { The Journal of } \\
\text { Politics } 75(1) \text { : } \\
\text { 183-97. }\end{array}$ & $\begin{array}{l}\text { institutional } \\
\text { design }\end{array}$ & $\mathrm{n}>4$ & $\begin{array}{l}\text { public } \\
\text { administration } \\
\text { (according to } \\
\text { journal) }\end{array}$ \\
\hline Johnson, T. & $2013 b$ & $\begin{array}{l}\text { 'Looking beyond states: openings } \\
\text { for international bureaucrats to } \\
\text { enter the institutional design } \\
\text { process' }\end{array}$ & $\begin{array}{l}\text { The Review of } \\
\text { International } \\
\text { Organizations } \\
\text { 8(4): 499-519. }\end{array}$ & $\begin{array}{l}\text { institutional } \\
\text { design }\end{array}$ & $\mathrm{n}>4$ & $\begin{array}{l}\text { international } \\
\text { relations }\end{array}$ \\
\hline Johnson, $\mathrm{T}$. & 2014 & $\begin{array}{l}\text { Organizational Progeny: Why } \\
\text { Governments are Losing Control } \\
\text { over the Proliferating Structures of } \\
\text { Global Governance }\end{array}$ & $\begin{array}{l}\text { Oxford: Oxford } \\
\text { University Press. }\end{array}$ & $\begin{array}{l}\text { policy } \\
\text { implementation }\end{array}$ & $\mathrm{n}>4$ & $\begin{array}{l}\text { international } \\
\text { relations }\end{array}$ \\
\hline $\begin{array}{l}\text { Johnson, T. and } \\
\text { Urpelainen, J. }\end{array}$ & 2014 & $\begin{array}{l}\text { 'International bureaucrats and the } \\
\text { formation of intergovernmental } \\
\text { organizations: institutional design } \\
\text { discretion sweetens the pot' }\end{array}$ & $\begin{array}{l}\text { International } \\
\text { Organization } \\
\text { 68(1): 177-209. }\end{array}$ & $\begin{array}{l}\text { institutional } \\
\text { design }\end{array}$ & $\mathrm{n}>4$ & $\begin{array}{l}\text { international } \\
\text { relations }\end{array}$ \\
\hline Jordan, R.S. & 1991 & $\begin{array}{l}\text { 'The fluctuating fortunes of the } \\
\text { United Nations International Civil } \\
\text { Service: hostage to politics or } \\
\text { undeservedly criticized?' }\end{array}$ & $\begin{array}{l}\text { Public } \\
\text { Administration } \\
\text { Review 51(4): } \\
\text { 353-57. }\end{array}$ & $\begin{array}{l}\text { institutional } \\
\text { design }\end{array}$ & UN & $\begin{array}{l}\text { public } \\
\text { administration }\end{array}$ \\
\hline Jordan, R.S. & 1981 & $\begin{array}{l}\text { 'What has happened to our } \\
\text { international civil service? the case } \\
\text { of the United Nations' }\end{array}$ & $\begin{array}{l}\text { Public } \\
\text { Administration } \\
\text { Review 41(2): } \\
\text { 236-45. }\end{array}$ & $\begin{array}{l}\text { institutional } \\
\text { design }\end{array}$ & UN & $\begin{array}{l}\text { public } \\
\text { administration }\end{array}$ \\
\hline $\begin{array}{l}\text { Juncos, A.E. and } \\
\text { Pomorska, K. }\end{array}$ & 2013 & $\begin{array}{l}\text { "In the face of adversity": } \\
\text { explaining the attitudes of EEAS } \\
\text { officials vis-à-vis the new service' }\end{array}$ & $\begin{array}{l}\text { Journal of } \\
\text { European Public } \\
\text { Policy 20(9): } \\
1332-49 .\end{array}$ & individual level & $\mathrm{EU}$ & $\begin{array}{l}\text { public } \\
\text { administration }\end{array}$ \\
\hline $\begin{array}{l}\text { Kanninen, T. and } \\
\text { Piiparinen, T. }\end{array}$ & 2014 & $\begin{array}{l}\text { 'Why bureaucracies matter in the } \\
\text { global age: a post-Weberian } \\
\text { explanation with the case study of } \\
\text { preparing and implementing the } \\
\text { United Nations' An Agenda for } \\
\text { Peace' }\end{array}$ & $\begin{array}{l}\text { International } \\
\text { Relations 28(1): } \\
46-66 .\end{array}$ & $\begin{array}{l}\text { institutional } \\
\text { design }\end{array}$ & UN & $\begin{array}{l}\text { international } \\
\text { relations }\end{array}$ \\
\hline Katz, A. & 2008 & $\begin{array}{l}\text { 'The independence of international } \\
\text { civil servants during the neoliberal } \\
\text { decades: implications of the work } \\
\text { stoppage involving } 700 \text { staff of the } \\
\text { World Health Organization in } \\
\text { November 2005' }\end{array}$ & $\begin{array}{l}\text { International } \\
\text { Journal of Health } \\
\text { Services } 38(1): \\
161-82 .\end{array}$ & $\begin{array}{l}\text { institutional } \\
\text { design }\end{array}$ & WHO & other discipline \\
\hline Kleine, M. & 2013 & $\begin{array}{l}\text { Informal Governance in the } \\
\text { European Union: How } \\
\text { Governments Make International } \\
\text { Organizations Work }\end{array}$ & $\begin{array}{l}\text { Ithaca (NY): } \\
\text { Cornell University } \\
\text { Press. }\end{array}$ & $\begin{array}{l}\text { policy } \\
\text { formulation }\end{array}$ & EU & $\begin{array}{l}\text { international } \\
\text { relations }\end{array}$ \\
\hline
\end{tabular}




\begin{tabular}{|c|c|c|c|c|c|c|}
\hline Korneev, O. & 2013 & $\begin{array}{l}\text { 'Exchanging knowledge, } \\
\text { enhancing capacities, developing } \\
\text { mechanisms: IOM's role in the } \\
\text { implementation of the EU-Russia } \\
\text { readmission agreement' }\end{array}$ & $\begin{array}{l}\text { Journal of Ethnic } \\
\text { and Migration } \\
\text { Studies } 40(6) \text { : } \\
888-904 .\end{array}$ & $\begin{array}{l}\text { policy } \\
\text { implementation }\end{array}$ & EU, IOM & other discipline \\
\hline $\begin{array}{l}\text { Weaver, C. and } \\
\text { Leiteritz, R.J. }\end{array}$ & 2005 & $\begin{array}{l}\text { "'Our poverty is a world full of } \\
\text { dreams:" reforming the World } \\
\text { Bank' }\end{array}$ & $\begin{array}{l}\text { Global } \\
\text { Governance 11(3): } \\
\text { 369-88. }\end{array}$ & $\begin{array}{l}\text { institutional } \\
\text { design }\end{array}$ & World Bank & $\begin{array}{l}\text { international } \\
\text { relations }\end{array}$ \\
\hline $\begin{array}{l}\text { Marcussen, M. } \\
\text { and Trondal, J. }\end{array}$ & 2011 & $\begin{array}{l}\text { 'The OECD civil servant: caught } \\
\text { between Scylla and Charybdis' }\end{array}$ & $\begin{array}{l}\text { Review of } \\
\text { International } \\
\text { Political Economy } \\
\text { 18(5): 592-621. }\end{array}$ & individual level & OECD & $\begin{array}{l}\text { international } \\
\text { relations }\end{array}$ \\
\hline Mathiason, J. & 2007 & $\begin{array}{l}\text { Invisible Governance: } \\
\text { International Secretariats in } \\
\text { Global Politics }\end{array}$ & $\begin{array}{l}\text { Boulder (CO): } \\
\text { Kumarian Press. }\end{array}$ & $\begin{array}{l}\text { policy } \\
\text { implementation }\end{array}$ & $\begin{array}{l}\mathrm{n}>4 \text { (World } \\
\text { Bank, UN, } \\
\text { UNICEF, } \\
\text { UNDP, WFP) }\end{array}$ & $\begin{array}{l}\text { international } \\
\text { relations }\end{array}$ \\
\hline McLaren, R. & 1980 & $\begin{array}{l}\text { Civil Servants and Public Policy: } \\
\text { A Comparative Study of } \\
\text { International Secretariats }\end{array}$ & $\begin{array}{l}\text { Waterloo }(\mathrm{ON}) \text { : } \\
\text { Wilfrid Laurier } \\
\text { University Press. }\end{array}$ & $\begin{array}{l}\text { policy } \\
\text { formulation }\end{array}$ & $\begin{array}{l}\mathrm{n}>4 \text { (IMO, } \\
\text { ITU, UPU, } \\
\text { WMO, } \\
\text { ICAO) }\end{array}$ & $\begin{array}{l}\text { public } \\
\text { administration }\end{array}$ \\
\hline $\begin{array}{l}\text { Morgan, C. and } \\
\text { Shahjahan, R.A. }\end{array}$ & 2014 & $\begin{array}{l}\text { 'The legitimation of OECD's } \\
\text { global educational governance: } \\
\text { examining PISA and AHELO test } \\
\text { production' }\end{array}$ & $\begin{array}{l}\text { Comparative } \\
\text { Education 50(2): } \\
192-205 .\end{array}$ & $\begin{array}{l}\text { policy } \\
\text { implementation }\end{array}$ & OECD & other discipline \\
\hline Mouritzen, $\mathrm{H}$. & 1990 & $\begin{array}{l}\text { The International Civil Service: A } \\
\text { Study of Bureaucracy: } \\
\text { International Organizations }\end{array}$ & $\begin{array}{l}\text { Aldershot: } \\
\text { Dartmouth. }\end{array}$ & $\begin{array}{l}\text { institutional } \\
\text { design }\end{array}$ & NATO & $\begin{array}{l}\text { international } \\
\text { relations }\end{array}$ \\
\hline Nay, O. & 2012 & $\begin{array}{l}\text { 'How do policy ideas spread } \\
\text { among international } \\
\text { administrations? policy } \\
\text { entrepreneurs and bureaucratic } \\
\text { influence in the UN response to } \\
\text { AIDS' }\end{array}$ & $\begin{array}{l}\text { Journal of Public } \\
\text { Policy 32(1): 53- } \\
76 .\end{array}$ & $\begin{array}{l}\text { policy } \\
\text { implementation }\end{array}$ & UNAIDS & $\begin{array}{l}\text { public } \\
\text { administration }\end{array}$ \\
\hline Nay, O. & 2011 & $\begin{array}{l}\text { 'What drives reforms in } \\
\text { international organizations? } \\
\text { external pressure and bureaucratic } \\
\text { entrepreneurs in the UN response } \\
\text { to AIDS' }\end{array}$ & $\begin{array}{l}\text { Governance 24(4): } \\
689-712 .\end{array}$ & $\begin{array}{l}\text { institutional } \\
\text { design }\end{array}$ & UNAIDS & $\begin{array}{l}\text { public } \\
\text { administration }\end{array}$ \\
\hline Nenadović, M. & 2010 & $\begin{array}{l}\text { 'An uneasy symbiosis: the impact } \\
\text { of international administrations on } \\
\text { political parties in post-conflict } \\
\text { countries' }\end{array}$ & $\begin{array}{l}\text { Democratization } \\
17(6): 1153-75 .\end{array}$ & $\begin{array}{l}\text { policy } \\
\text { implementation }\end{array}$ & UN & $\begin{array}{l}\text { public } \\
\text { administration }\end{array}$ \\
\hline Oestreich, J.E. & 2007 & $\begin{array}{l}\text { Power and Principle: Human } \\
\text { Rights Programming in } \\
\text { International Organizations }\end{array}$ & $\begin{array}{l}\text { Washington D.C.: } \\
\text { Georgetown } \\
\text { University Press. }\end{array}$ & $\begin{array}{l}\text { policy } \\
\text { formulation }\end{array}$ & $\begin{array}{l}\text { World Bank, } \\
\text { WHO, } \\
\text { UNICEF }\end{array}$ & $\begin{array}{l}\text { public } \\
\text { administration }\end{array}$ \\
\hline Park, S. & 2010 & $\begin{array}{l}\text { World Bank Group Interactions } \\
\text { with Environmentalists: Changing } \\
\text { International Organisation } \\
\text { Identities }\end{array}$ & $\begin{array}{l}\text { Manchester: } \\
\text { Manchester } \\
\text { University Press. }\end{array}$ & $\begin{array}{l}\text { policy } \\
\text { formulation }\end{array}$ & World Bank & $\begin{array}{l}\text { international } \\
\text { relations }\end{array}$ \\
\hline Piiparinen, $\mathrm{T}$. & 2009 & $\begin{array}{l}\text { The Transformation of UN } \\
\text { Conflict Management: Producing } \\
\text { Images of Genocide from Rwanda } \\
\text { to Darfur and Beyond }\end{array}$ & $\begin{array}{l}\text { London: } \\
\text { Routledge. }\end{array}$ & $\begin{array}{l}\text { policy } \\
\text { implementation }\end{array}$ & UN & $\begin{array}{l}\text { international } \\
\text { relations }\end{array}$ \\
\hline $\begin{array}{l}\text { Puntigliano, A.R. } \\
\text { and Appelqvist, } \\
\text { Ö. }\end{array}$ & 2011 & $\begin{array}{l}\text { 'Prebisch and Myrdal: } \\
\text { development economics in the } \\
\text { core and on the periphery' }\end{array}$ & $\begin{array}{l}\text { Journal of Global } \\
\text { History 6(1): 29- } \\
52 \text {. }\end{array}$ & $\begin{array}{l}\text { policy } \\
\text { implementation }\end{array}$ & UN & other discipline \\
\hline
\end{tabular}




\begin{tabular}{|c|c|c|c|c|c|c|}
\hline $\begin{array}{l}\text { Reymond, H. and } \\
\text { Mailick, S. }\end{array}$ & 1986 & $\begin{array}{l}\text { 'The international civil service } \\
\text { revisited' }\end{array}$ & $\begin{array}{l}\text { Public } \\
\text { Administration } \\
\text { Review 46(2): } \\
135-43 .\end{array}$ & $\begin{array}{l}\text { institutional } \\
\text { design }\end{array}$ & UN & $\begin{array}{l}\text { public } \\
\text { administration }\end{array}$ \\
\hline Sarfaty, G. & 2012 & $\begin{array}{l}\text { Values in Translation: Human } \\
\text { Rights and the Culture of the } \\
\text { World Bank }\end{array}$ & $\begin{array}{l}\text { Stanford: Stanford } \\
\text { University Press. }\end{array}$ & $\begin{array}{l}\text { institutional } \\
\text { design }\end{array}$ & World Bank & $\begin{array}{l}\text { public } \\
\text { administration }\end{array}$ \\
\hline Schroeder, M. & 2013 & $\begin{array}{l}\text { 'The politics of change: the } \\
\text { evolution of UN electoral services, } \\
\text { 1989-2006' }\end{array}$ & $\begin{array}{l}\text { Global } \\
\text { Governance 19(2): } \\
\text { 207-26. }\end{array}$ & $\begin{array}{l}\text { policy } \\
\text { formulation }\end{array}$ & UN & $\begin{array}{l}\text { international } \\
\text { relations }\end{array}$ \\
\hline Selcer, P. & 2012 & $\begin{array}{l}\text { 'Beyond the cephalic index: } \\
\text { negotiating politics to produce } \\
\text { UNESCO's scientific statements } \\
\text { on race' }\end{array}$ & $\begin{array}{l}\text { Current } \\
\text { Anthropology } \\
\text { 53(S5): 173-84. }\end{array}$ & $\begin{array}{l}\text { policy } \\
\text { formulation }\end{array}$ & UNESCO & other discipline \\
\hline Senarclens, P. d. & 2001 & $\begin{array}{l}\text { 'International organisations and } \\
\text { the challenges of globalisation' }\end{array}$ & $\begin{array}{l}\text { International } \\
\text { Social Science } \\
\text { Journal 53(170): } \\
509-22 .\end{array}$ & $\begin{array}{l}\text { policy } \\
\text { implementation }\end{array}$ & $\begin{array}{l}\text { several } \\
\text { examples }\end{array}$ & other discipline \\
\hline Sharma, P.A. & 2011 & $\begin{array}{l}\text { 'Bureaucratic imperatives and } \\
\text { policy outcomes: the origins of } \\
\text { World Bank structural adjustment } \\
\text { lending' }\end{array}$ & $\begin{array}{l}\text { Review of } \\
\text { International } \\
\text { Political Economy } \\
\text { 20(4): 667-86. }\end{array}$ & $\begin{array}{l}\text { policy } \\
\text { formulation }\end{array}$ & $\begin{array}{l}\text { IMF, World } \\
\text { Bank }\end{array}$ & $\begin{array}{l}\text { international } \\
\text { relations }\end{array}$ \\
\hline Shepard, T. & 2011 & $\begin{array}{l}\text { 'Algeria, France, Mexico, } \\
\text { UNESCO: a transnational history } \\
\text { of anti-racism and decolonization, } \\
\text { 1932-1962' }\end{array}$ & $\begin{array}{l}\text { Journal of Global } \\
\text { History 6(2): 273- } \\
97 .\end{array}$ & $\begin{array}{l}\text { policy } \\
\text { implementation }\end{array}$ & $\begin{array}{l}\text { UN, } \\
\text { UNESCO }\end{array}$ & other discipline \\
\hline Stone, R.W. & 2011 & $\begin{array}{l}\text { Controlling Institutions: } \\
\text { International Organizations and } \\
\text { the Global Economy }\end{array}$ & $\begin{array}{l}\text { Cambridge: } \\
\text { Cambridge } \\
\text { University Press. }\end{array}$ & $\begin{array}{l}\text { policy } \\
\text { implementation }\end{array}$ & $\begin{array}{l}\text { EU, IMF, } \\
\text { WTO }\end{array}$ & $\begin{array}{l}\text { international } \\
\text { relations }\end{array}$ \\
\hline Strange, S. & 1996 & $\begin{array}{l}\text { The Retreat of the State: The } \\
\text { Diffusion of Power in the World } \\
\text { Economy }\end{array}$ & $\begin{array}{l}\text { Cambridge: } \\
\text { Cambridge } \\
\text { University Press. }\end{array}$ & $\begin{array}{l}\text { policy } \\
\text { implementation }\end{array}$ & $\begin{array}{l}\text { IMF, Wold } \\
\text { Bank, EU }\end{array}$ & $\begin{array}{l}\text { international } \\
\text { relations }\end{array}$ \\
\hline Trondal, J. & 2011 & $\begin{array}{l}\text { 'Bureaucratic structure and } \\
\text { administrative behaviour: lessons } \\
\text { from international bureaucracies' }\end{array}$ & $\begin{array}{l}\text { West European } \\
\text { Politics 34(4): } \\
795-818 .\end{array}$ & individual level & $\begin{array}{l}\text { EU, OECD, } \\
\text { WTO }\end{array}$ & $\begin{array}{l}\text { public } \\
\text { administration }\end{array}$ \\
\hline $\begin{array}{l}\text { Trondal, J., } \\
\text { Marcussen, M., } \\
\text { Larsson, T. and } \\
\text { Veggeland, F. }\end{array}$ & 2010 & $\begin{array}{l}\text { Unpacking International } \\
\text { Organisations: The Dynamics of } \\
\text { Compound Bureaucracies }\end{array}$ & $\begin{array}{l}\text { Manchester: } \\
\text { Manchester } \\
\text { University Press. }\end{array}$ & $\begin{array}{l}\text { policy } \\
\text { implementation }\end{array}$ & $\begin{array}{l}\text { EU, OECD, } \\
\text { WTO }\end{array}$ & $\begin{array}{l}\text { public } \\
\text { administration }\end{array}$ \\
\hline Udom, U.E. & 2003 & $\begin{array}{l}\text { 'The international civil service: } \\
\text { historical development and } \\
\text { potential for the } 21 \text { st century' }\end{array}$ & $\begin{array}{l}\text { Public Personnel } \\
\text { Management } \\
\text { 32(1): 49-50. }\end{array}$ & $\begin{array}{l}\text { institutional } \\
\text { design }\end{array}$ & UN & $\begin{array}{l}\text { public } \\
\text { administration }\end{array}$ \\
\hline $\begin{array}{l}\text { Vaubel, R., } \\
\text { Dreher, A. and } \\
\text { Soylu, U. }\end{array}$ & 2007 & $\begin{array}{l}\text { 'Staff growth in international } \\
\text { organizations: a principal-agent } \\
\text { problem? an empirical analysis' }\end{array}$ & $\begin{array}{l}\text { Public Choice } \\
\text { 133(3): 275-95. }\end{array}$ & $\begin{array}{l}\text { institutional } \\
\text { design }\end{array}$ & $\mathrm{n}>4$ & $\begin{array}{l}\text { public } \\
\text { administration }\end{array}$ \\
\hline $\begin{array}{l}\text { Verweij, M. and } \\
\text { Josling, T. }\end{array}$ & 2003 & $\begin{array}{l}\text { 'Special issue: deliberately } \\
\text { democratizing multilateral } \\
\text { organization' }\end{array}$ & $\begin{array}{l}\text { Governance 16(1): } \\
1-21 \text {. }\end{array}$ & $\begin{array}{l}\text { institutional } \\
\text { design }\end{array}$ & $\begin{array}{l}\text { IMF, World } \\
\text { Bank, WTO }\end{array}$ & $\begin{array}{l}\text { public } \\
\text { administration }\end{array}$ \\
\hline Vetterlein, A. & 2012 & $\begin{array}{l}\text { 'Seeing like the World Bank on } \\
\text { poverty' }\end{array}$ & $\begin{array}{l}\text { New Political } \\
\text { Economy 17(1): } \\
35-58 .\end{array}$ & $\begin{array}{l}\text { policy } \\
\text { formulation }\end{array}$ & World Bank & $\begin{array}{l}\text { international } \\
\text { relations }\end{array}$ \\
\hline Vilella, G. & 1999 & $\begin{array}{l}\text { 'Importance and role of } \\
\text { supranational administration: the } \\
\text { case of European Union } \\
\text { administration' }\end{array}$ & $\begin{array}{l}\text { International } \\
\text { Review of } \\
\text { Administrative }\end{array}$ & $\begin{array}{l}\text { policy } \\
\text { implementation }\end{array}$ & $\mathrm{EU}$ & $\begin{array}{l}\text { public } \\
\text { administration }\end{array}$ \\
\hline
\end{tabular}




\begin{tabular}{|c|c|c|c|c|c|c|}
\hline & & & $\begin{array}{l}\text { Sciences } 65(2): \\
211-19 .\end{array}$ & & & \\
\hline Warning, M.J. & 2009 & $\begin{array}{l}\text { Transnational Public Governance: } \\
\text { Networks, Law and Legitimacy }\end{array}$ & $\begin{array}{l}\text { Basingstoke: } \\
\text { Palgrave } \\
\text { Macmillan. }\end{array}$ & $\begin{array}{l}\text { policy } \\
\text { implementation }\end{array}$ & EU & $\begin{array}{l}\text { international } \\
\text { relations }\end{array}$ \\
\hline Weiss, T.G. & 2010 & $\begin{array}{l}\text { 'The John W. Holmes lecture: } \\
\text { reinvigorating the international } \\
\text { civil service' }\end{array}$ & $\begin{array}{l}\text { Global } \\
\text { Governance 16(1): } \\
\text { 39-57. }\end{array}$ & $\begin{array}{l}\text { institutional } \\
\text { design }\end{array}$ & UN & $\begin{array}{l}\text { international } \\
\text { relations }\end{array}$ \\
\hline Weiss, T.G. & 2008 & $\begin{array}{l}\text { What's Wrong with the United } \\
\text { Nations and How to Fix It }\end{array}$ & $\begin{array}{l}\text { Cambridge: Polity } \\
\text { Press. }\end{array}$ & $\begin{array}{l}\text { institutional } \\
\text { design }\end{array}$ & $\mathrm{UN}$ & $\begin{array}{l}\text { international } \\
\text { relations }\end{array}$ \\
\hline Weiss, T.G. & 1982 & $\begin{array}{l}\text { 'International bureaucracy: the } \\
\text { myth and reality of the } \\
\text { international civil service' }\end{array}$ & $\begin{array}{l}\text { International } \\
\text { Affairs 58(2): 287- } \\
306 .\end{array}$ & $\begin{array}{l}\text { institutional } \\
\text { design }\end{array}$ & $\mathrm{UN}$ & $\begin{array}{l}\text { international } \\
\text { relations }\end{array}$ \\
\hline Weiss, T.G. & 1975 & $\begin{array}{l}\text { International Bureaucracy: An } \\
\text { Analysis of the Operation of } \\
\text { Functional and Global } \\
\text { International Secretariats }\end{array}$ & $\begin{array}{l}\text { Lexington (MA): } \\
\text { Lexington Books. }\end{array}$ & $\begin{array}{l}\text { institutional } \\
\text { design }\end{array}$ & UN, UNICEF & $\begin{array}{l}\text { international } \\
\text { relations }\end{array}$ \\
\hline $\begin{array}{l}\text { Widerberg, O. } \\
\text { and van } \\
\text { Laerhoven, F. }\end{array}$ & 2014 & $\begin{array}{l}\text { 'Measuring the autonomous } \\
\text { influence of an international } \\
\text { bureaucracy: the division for } \\
\text { sustainable development' }\end{array}$ & $\begin{array}{l}\text { International } \\
\text { Environmental } \\
\text { Agreements: } \\
\text { Politics, Law and } \\
\text { Economics 14(4): } \\
303-27 .\end{array}$ & $\begin{array}{l}\text { policy } \\
\text { implementation }\end{array}$ & UNFCCC & $\begin{array}{l}\text { public } \\
\text { administration }\end{array}$ \\
\hline Wille, A. & 2013 & $\begin{array}{l}\text { The Normalization of the } \\
\text { European Commission: Politics } \\
\text { and Bureaucracy in the EU } \\
\text { Executive }\end{array}$ & $\begin{array}{l}\text { Oxford: Oxford } \\
\text { University Press. }\end{array}$ & $\begin{array}{l}\text { institutional } \\
\text { design }\end{array}$ & EU & $\begin{array}{l}\text { public } \\
\text { administration }\end{array}$ \\
\hline Winslow, A. & 1970 & $\begin{array}{l}\text { 'Functions of an international } \\
\text { secretariat' }\end{array}$ & $\begin{array}{l}\text { Public } \\
\text { Administration } \\
\text { Review 30(3): } \\
\text { 211-16. }\end{array}$ & individual level & $\mathrm{UN}$ & $\begin{array}{l}\text { public } \\
\text { administration }\end{array}$ \\
\hline $\begin{array}{l}\mathrm{Xu}, \mathrm{Y} . \text { and } \\
\text { Weller, P. }\end{array}$ & 2004 & $\begin{array}{l}\text { The Governance of World Trade: } \\
\text { International Civil Servants and } \\
\text { the GATT/WTO }\end{array}$ & $\begin{array}{l}\text { Cheltenham, } \\
\text { Northampton } \\
\text { (MA): Edward } \\
\text { Elgar Publishing. }\end{array}$ & $\begin{array}{l}\text { policy } \\
\text { formulation }\end{array}$ & WTO & $\begin{array}{l}\text { public } \\
\text { administration }\end{array}$ \\
\hline $\begin{array}{l}\mathrm{Xu}, \mathrm{Y} . \text { and } \\
\text { Weller, P. }\end{array}$ & 2008 & $\begin{array}{l}\text { "To be but not to be seen": } \\
\text { exploring the impact of } \\
\text { international civil servants" }\end{array}$ & $\begin{array}{l}\text { Public } \\
\text { Administration } \\
\text { 86(1): 35-51. }\end{array}$ & $\begin{array}{l}\text { policy } \\
\text { implementation }\end{array}$ & WTO & $\begin{array}{l}\text { public } \\
\text { administration }\end{array}$ \\
\hline Zaum, D. & 2006 & $\begin{array}{l}\text { 'The authority of international } \\
\text { administrations in international } \\
\text { society' }\end{array}$ & $\begin{array}{l}\text { Review of } \\
\text { International } \\
\text { Studies 32(3): } \\
455-73 \text {. }\end{array}$ & $\begin{array}{l}\text { institutional } \\
\text { design }\end{array}$ & $\mathrm{UN}$ & $\begin{array}{l}\text { international } \\
\text { relations }\end{array}$ \\
\hline
\end{tabular}

